Reprod. Nutr. Dévelop., 1980, 20 (4 B), 1279-1284.

\title{
Influence de l'âge sur les sécrétions de suc gastrique ef d'électrolytes chez le veau préruminant
}

\author{
par P. GUILLOTEAU, R. TOULLEC, P. GARNOT *, P. MARTIN *, G. BRULE * \\ avec la collaboration technique de Margueriłe BEAUFILS, S. BOUSSION, Michèle FORMAL, J. LA- \\ REYNIE, Monique LESNE et Y. MANIS \\ Station de Recherches Zootechniques, I. N. R. A., \\ * Laboratoire de Recherches de Technologie Laitière, I. N. R. A., \\ 65, rue de Saint-Brieuc, 35042 Rennes Cedex, France
}

Summary. Effect of age on the secretion of gastric juice and ions in the preruminant calf.

Four preruminant Friesian calves were fitted with an innervated abomasal fundic pouch cut from the lesser curvature. They were fed twice daily a milk substitute containing only spray-dried skim-milk powder as protein source.

The daily outflow of gastric juice regularly increased from 400 to $1800 \mathrm{~g} /$ day between 2 and 16 weeks of age and did not change later. The daily outflow $/ 100 \mathrm{~kg}$ liveweight increased until 7 to 9 weeks of age and decreased later until 30 weeks (fig. 1). The $\mathrm{pH}$ of the secretion always ranged between 1 and 2, except during the days following surgery and when the calves were ill (fig. 2). Expressed in absolute terms, the total outflow of hydrogen, sodium, potassium and chloride ions increased with age ; this was mainly due to the increase in the outflow of gastric juice since the ion concentration showed a cyclic paftern with age (fig. 3). When expressed on a liveweight basis, the daily ion output alternatively showed increasing and decreasing periods, mainly in relation to changes in concentration, but no significant trend with age was observed (fig. 4).

\section{Introduction.}

Les sécrétions abomasales du veau préruminant n'ont généralement fait l'objet que d'études ponctuelles. Les auteurs se sont surtout intéressés au volume, à l'acidité et à l'activité pepsique du suc gastrique (Hill, 1968 ; Rusev, Petrov et Bocheva, 1971 ; Williams, Roy et Gillies, 1976). Le but de ce travail est de mesurer en continu, l'évolution de la sécrétion d'électrolytes (hydrogène, sodium, potassium ef chlore) en fonction de l'âge.

\section{Matériel ef méthodes.}

Quatre veaux mâles de race Frisonne sont munis d'une poche abomasale innervée découpée principalement dans la zone fundique, sur la petite courbure de la caillette (Guilloteau et Le Calve, 1977). Ils reçoivent un lait de remplacement conte- 
nant 25 p. 100 de protéines apportées en totalité par du lait écrémé ; cet aliment est distribué au seau deux fois par jour à raison de $58 \mathrm{~g}$ de matière sèche $/ \mathrm{kg}$ de poids vif $^{0,75} /$ jour. Le suc gastrique est collecté en permanence dans un sac en plastique. Après pesée, un échantillon journalier est prélevé et conservé à $-18^{\circ} \mathrm{C}$ jusqu'aux analyses. Celles-ci sont effectuées, pour les 3 premiers veaux, sur des échantillons représentatifs des sécrétions hebdomadaires, en excluant éventuellement les jours où les veaux ont présenté des troubles sanitaires. Certains échantillons journaliers sont également analysés séparément, afin d'apprécier l'amplifude des variations journalières. Le $\mathrm{pH}$ est mesuré à l'aide d'un $\mathrm{pH}$ mètre Radiometer PHM $28 \mathrm{D}$; les ions $\mathrm{H}^{+}$sont estimés en titrant l'acidité avec de la soude $0,1 \mathrm{~N}$ en présence de phénolphtaléine ; les ions $\mathrm{Na}^{+}$et $\mathrm{K}^{+}$sont dosés par spectrophotométrie d'absorption atomique et les ions $\mathrm{Cl}^{-}$par la méthode de Charpentier-Volhard.

\section{Résultats et discussion.}

Les quantités de suc gastrique sécrété augmentent régulièrement de 400 à $1800 \mathrm{~g} /$ jour jusqu'à l'âge de 16 semaines et se stabilisent ensuite. Les variations journalières sont importantes : ainsi, pour le veau $A$, les quantités sécrétées varient de 510 à $1200 \mathrm{~g}$ au cours de la quinzième semaine. Les quantités journalières rapporlées au poids vif de l'animal augmentent jusqu'à 7 à 9 semaines et diminuent ensuite jusqu'à 30 semaines (fig. 1). Williams, Roy et Gillies (1976) indiquent que la sécrétion

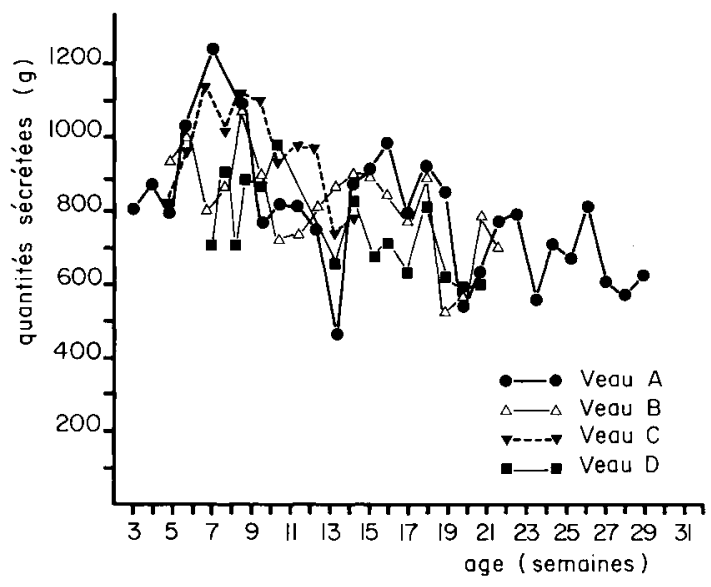

FIG. 1. - Evolution avec l'âge des quantités journalières de suc gastrique sécrété par $100 \mathrm{~kg}$ de poids vif (moyennes hebdomadaires).

d'une poche abomasale varie de 7,3 à $13,8 \mathrm{ml} / \mathrm{kg} P \mathrm{PV}^{0,75}$ pendant $8 \mathrm{~h}$, ce qui correspondrait à une quantité de 720 à $1700 \mathrm{~g}$ de suc gastrique $/ 100 \mathrm{~kg}$ de poids vif/jour. Ces valeurs sont un peu plus élevées que les nôtres mais elles doivent varier en fonction de la technique chirurgicale adoptée. La surface de la poche abomasale des veaux utilisés représente $1 / 4$ à $1 / 8$ de la surface totale de la caillette (Guilloteau et Le Calve, 1977) ; la quantité minimale de suc sécrété serait donc comprise entre 2,4 
TABLEAU 1

Composition des sucs gastriques collectés (méq/l) :

moyennes des échantillons hebdomadaires (écarts types des échantillons)

\begin{tabular}{lcccccc}
\hline & Veau & $\begin{array}{c}\text { Nombre de } \\
\text { mesures }\end{array}$ & $\mathrm{Cl}$ & $\mathrm{H}^{+}$ & $\mathrm{Na}^{+}$ & $\mathrm{K}^{+}$ \\
\hline A $\ldots \ldots \ldots$ & 30 & $87(21) \mathrm{a}$ & $46(10)$ & $87(21) \mathrm{bd}$ & $9(2)$ \\
\hline B $\ldots \ldots \ldots$ & 13 & $72(32) \mathrm{c}$ & $49(13)$ & $49(9) \mathrm{ab}$ & $9(2)$ \\
\hline C $\ldots \ldots \ldots$ & 18 & $145(75) \mathrm{ac}$ & $47(10)$ & $75(12) \mathrm{ad}$ & $8(1)$ \\
\hline
\end{tabular}

$a, b, c, d: 2$ valeurs d'une même colonne accompagnées d'une même lettre sont significativement différentes ( $a$ ef $b: P<0,001, c: P<0,01$ et $d: P<0,05$ ).

nière différence peut s'expliquer par l'împrécision des méthodes utilisées, en particulier pour les ions $\mathrm{H}^{+}$, et par le fait qu'il n'a pas été tenu compte des ions $\mathrm{Ca}^{++}$et $\mathrm{Mg}^{++}$qui peuvent exister en faible concentration dans le suc gastrique (Hunt et Wan, 1967). La première différence indique que chez les veaux $A$ et $B$, la part des sécrétions non pariétales qui apportent des bicarbonates chez différentes espèces de mammifères (Hunt et Wan, 1967 ; Davenport, 1968 ; Konturek, 1974), serait bequcoup plus importante que chez le veau $C$, au moins dans le suc sécrété par la poche gastrique. Il est possible qu'il y ait de légères variations d'un animal à l'autre, dans l'emplacement anatomique de la poche. A notre connaissance, il n'a pas été publié de données con-

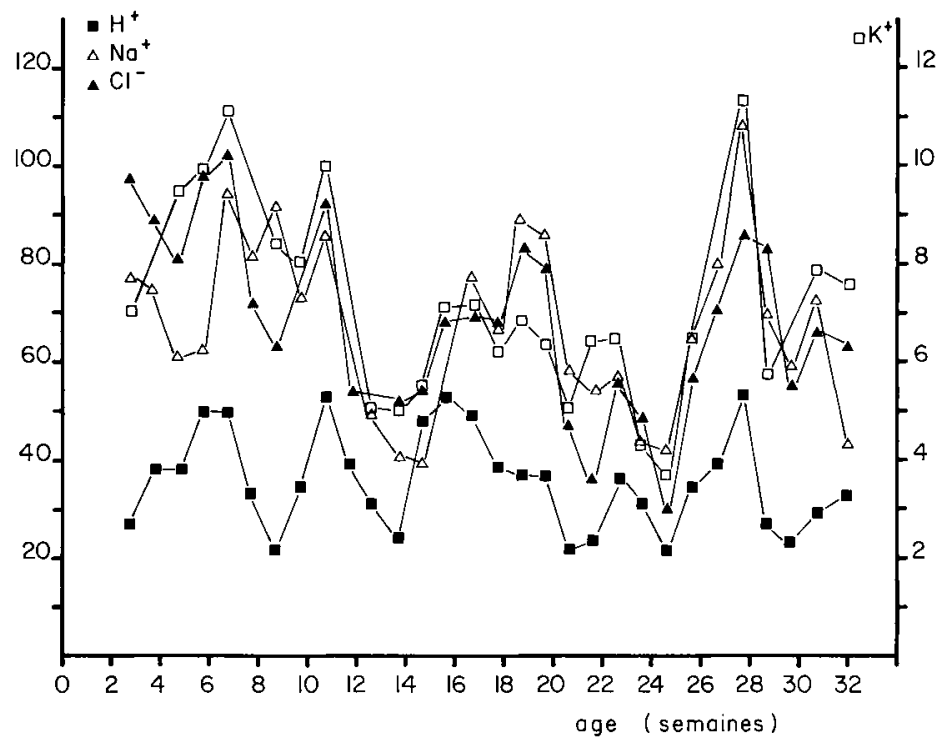

FIG. 3. - Evolution avec l'âge de la concentration du suc gastrique en électrolytes (méq/l) chez le Veau A (échantillons hebdomadaires). 
cernant simultanément les teneurs en $\mathrm{Cl}^{-}, \mathrm{H}^{+}, \mathrm{Na}^{+}$et $\mathrm{K}^{+}$dans le suc sécrété en $24 \mathrm{~h}$ par une poche gastrique de veau préruminant. Williams, Roy et Gillies (1976), analysant des sucs gastriques représentatifs des $8 \mathrm{~h}$ qui suivent le repas du matin, obtiennent des concentrations du même ordre de grandeur que nous en $\mathrm{Cl}^{-}(117 \mathrm{méq} / \mathrm{l})$ et en $\mathrm{K}^{+}(12 \mathrm{méq} / \mathrm{l})$ mais plus faibles en $\mathrm{Na}^{+}$(35 méq/l) ; dans d'autres échantillons, analysés heure par heure, ces auteurs trouvent davantage d'acidité titrable que nous (de 54 à 92 méq/l).

Les quantités totales d'électrolyłes sécrétés augmentent avec l'âge ; cela est principalement dû à un accroissement du volume de suc sécrété puisaue les concentrations en électrolytes évoluent de manière cyclique avec l'âge (fig. 3). Les fluctuations journalières sont importantes : ainsi, chez le veau $A$, au cours de la quinzième semaine, les quantités d'ions $\mathrm{Cl}^{-}, \mathrm{H}^{+}, \mathrm{Na}^{+}$et $\mathrm{K}^{+}$sécrétés varient respectivement de 49 à 166 , de 36 à 97, de 41 à 111 et de 5 à 13 méq/jour. Rapportées au poids vif (fig. 4) ou au poids métabolique, les quantités journalières d'électrolyłes sécrétés présentent une alternance de périodes d'augmentation et de diminution liées principalement aux variations des concentrations, sans qu'il soit possible de mettre en évidence une autre tendance générale.

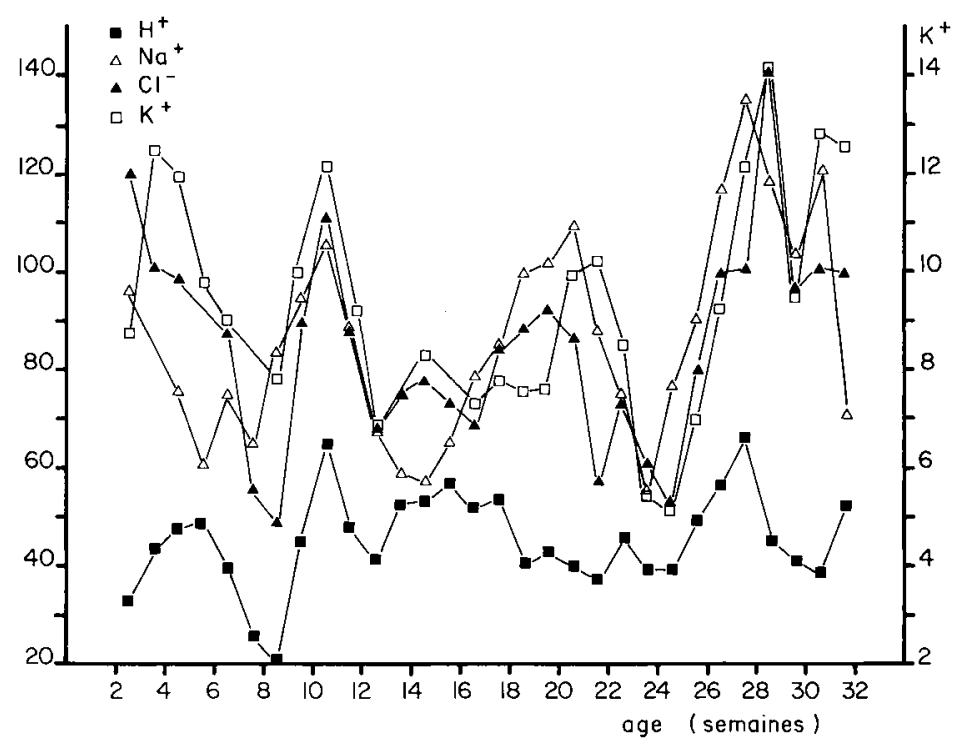

FiG. 4. - Evolution avec l'âge des quantités d'électrolytes (méq/jour/100 kg de poids vif) sécrétés chez le Veau $A$ (échantillons hebdomadaires).

Chez des veaux porteurs d'une double canule du duodénum, placée après l'arrivée de la bile, le suc pancréatique étant dérivé, Ternouth, Roy ef Shotton (1976) observent que les sécrétions de $\mathrm{K}^{+}$et $\mathrm{Cl}^{-}$, rapportées au poids métabolique, ne varient pas significativement entre 7,24 et 63 jours ; en revanche, la sécrétion de $\mathrm{H}^{+}$ double entre 7 et 24 jours et triple entre 24 et 63 jours, alors que celle de $\mathrm{Na}^{+}$diminue entre 7 et 24 jours. Cependant, l'effet des variations cycliques éventuelles ne peut pas 
être mis en évidence par des mesures ponctuelles. Il est en fout cas difficile de comparer ces résultats aux nôtres, étant donné l'influence des apports salivaires et biliaires (Sasaki, 1968 ; Debarre et al., 1979), ainsi que l'effet tampon des protéines alimentaires.

En conclusion, dans la mesure où la poche est représentative de l'ensemble de la caillette, les variations des sécrétions journalières d'électrolytes en fonction de l'âge sont moins déterminées par les changements de volume du suc que par ceux observés dans leurs concentrations respectives. Les quantités journalières sécrétées augmentent avec l'âge en valeur absolue ; cependant, si on les rapporte au poids vif de l'animal des variations cycliques sont observées mais aucune évolution systématique n'apparaît. Les ions $\mathrm{Na}^{+}$et $\mathrm{Cl}^{-}$suivent pour chaque veau une évolution comparable ef sont sécrétés en quantités voisines, la production d'ions $\mathrm{H}^{+}$est plus faible ef moins variable ef celle des ions $\mathrm{K}^{+}$suit la même évolution que les 2 premiers électrolytes mais est 10 fois plus faible.

Journées Ingestion-Digestion-Absorption de l'Association française de Nutrition, Paris, 15-16 novembre 1979.

\section{Références}

DAVENPORT H. W., 1968. Physiologie de l'appareil digestif, 94-120, Masson, Paris.

DEBARRE M., THIVEND P., LEFAIVRE J., TOULLEC R., 1979. Biliary secretion in the preruminant calf. Ann. Rech. vét., 10, 425-427.

GUILLOTEAU P., LE CALVE J. L., 1977. Technique de réalisation d'une poche abomasale chez le veau en vue de l'obtention de suc gastrique pur. Ann. Biol. anim. Bioch. Biophys., 17, 1047-1060.

HILL K. J., 1968. Abomasal function. In CODE F. C., HEIDEL W., Handbook of physiology, Sect. 6 : Alimentary Canal, Vol. V. Bile, digestion, ruminal physiology, 2747-2759, Williams and Wilkins, Baltimore.

HUNT J. N., WAN B., 1967. Electrolytes of mammalian gastric juice. In CODE F. C., HEIDEL W., Handbook of physiology, sect. 6 : Alimentary canal, vol. II. Secretion, 781-804, Williams and Wilkins, Baltimore.

KONTUREK S. J., 1974. Gastric secretion, 227-264. In JACOBSON E. D., SHANBOUR L. L., Gastroinfestinal physiology, Butterworth, Londres.

ROY J. H. B., STOBO I. J. F., 1975. Nutrition of the preruminant calf, 30-48. In Mc DONALD J. W., WARNER A. C. I., Digestion and metabolism in the ruminant. Proc. IVth int. Symp. on ruminant physiology, Univ. of New England Publ. Unit., Armidale.

RUSEV V., PETROV A., BOCHEVA E., 1971. Peculiarities of rennet secretion in nursing calves in connection with the manner of feeding and milk temperature (en russe). Nautsh. Trud. Zootech. Fac., 22, 145-156.

SASAKI Y., 1968. Development of mixed saliva secretion in young calves. Jap. J. zootech. Sci., 39, 333-340.

TERNOUTH J. H., ROY J. H. B., SHOTTON S. M., 1976. Concurrent studies of the flow of digesta in the duodenum and of exocrine pancreatic secretion of calves. 4. The effect of age. Br. J. Nutr., 36, 523-535.

WILLIAMS V. J., ROY J. H. B., GILLIES C. M., 1976. Milk substitute diet composition and abomasal secretion in the calf. Br. J. Nutr., 36, 317-335. 